\title{
Relationship Between Cognitive Function and Dysphagia After Stroke
}

\author{
Soo Yung Jo, MD, Jeong-Won Hwang, MD, Sung-Bom Pyun, MD, PhD
}

Department of Physical Medicine and Rehabilitation, Korea University College of Medicine, Seoul, Korea

\begin{abstract}
Objective To investigate the characteristics of cognitive deficits in patients with post-stroke dysphagia, and to analyze the relationships between cognitive dysfunction and severity of dysphagia in supratentorial stroke.

Methods A total of 55 patients with first-ever supratentorial lesion stroke were enrolled retrospectively, within 3 months of onset. We rated dysphagia from 0 (normal) to 4 (severe) using the dysphagia severity scale (DSS) through clinical examinations and videofluoroscopic swallowing studies (VFSS). The subjects were classified either as non-dysphagic (scale 0 ) or dysphagic (scale 1 to 4 ). We compared general characteristics, stroke severity and the functional scores of the two groups. We then performed comprehensive cognitive function tests and investigated the differences in cognitive performance between the two groups, and analyzed the correlation between cognitive test scores, DSS, and parameters of oral and pharyngeal phase.

Results Fugl-Meyer motor assessment, the Berg Balance Scale, and the Korean version of the Modified Barthel Index showed significant differences between the two groups. Cognitive test scores for the dysphagia group were significantly lower than the non-dysphagia group. Significant correlations were shown between dysphagia severity and certain cognitive subtest scores: visual span backward $(\mathrm{p}=0.039)$, trail making tests $\mathrm{A}(\mathrm{p}=0.042)$ and $\mathrm{B}$ $(\mathrm{p}=0.002)$, and Raven progressive matrices $(\mathrm{p}=0.002)$. The presence of dysphagia was also significantly correlated with cognitive subtests, in particular for visual attention and executive attention (odds ratio [OR]=1.009; 95\% confidence interval $[\mathrm{CI}], 1.002-1.016 ; \mathrm{p}=0.017)$. Parameters of premature loss were also significantly correlated with the same subtests (OR=1.009; 95\% CI, 1.002-1.016; $\mathrm{p}=0.017$ ).

Conclusion Our results suggest that cognitive function is associated with the presence and severity of post-stroke dysphagia. Above all, visual attention and executive functions may have meaningful influence on the oral phase of swallowing in stroke patients with supratentorial lesions.
\end{abstract}

Keywords Deglutition disorders, Stroke, Cognition, Attention, Executive function

Received October 12, 2016; Accepted October 24, 2016

Corresponding author: Sung-Bom Pyun

Department of Physical Medicine and Rehabilitation, Korea University Anam Hospital, 73 Inchon-ro, Seongbuk-gu, Seoul 02841, Korea. Tel: +82-2-9206471, Fax: +82-2-929-9951, E-mail: rmpyun@korea.ac.kr

ORCID: Soo Yung Jo (http://orcid.org/0000-0002-0516-2725); Jeong-Won Hwang (http://orcid.org/0000-0002-5138-7538); Sung-Bom Pyun (http:// orcid.org/0000-0002-3471-9692).

(c) This is an open-access article distributed under the terms of the Creative Commons Attribution Non-Commercial License (http://creativecommons.org/ licenses/by-nc/4.0) which permits unrestricted noncommercial use, distribution, and reproduction in any medium, provided the original work is properly cited. Copyright $\odot 2017$ by Korean Academy of Rehabilitation Medicine 


\section{INTRODUCTION}

Dysphagia is a common symptom in stroke patients with up to $50 \%-70 \%$ of all cases experiencing swallowing problems in their acute stage [1]. Dysphagia can cause deterioration in activities of daily living (ADL) and quality of life, resulting in malnutrition, dehydration, aspiration pneumonia, and increased mortality [2]. Therefore, it is important to assess the presence of dysphagia in the initial stages of post-stroke rehabilitation.

Although dysphagia is a major source of disability in stroke patients, its neurobiological basis is largely unknown. It has been established that both cerebral cortical infarctions as well as brain stem infarctions can cause dysphagia, however, it is still controversial as to which of the two hemispheres plays a more important role in swallowing, and which injured hemisphere is more likely to cause the specific patterns of dysphagia seen in stroke patients [3-5]. Daniels et al. [6] proposed that the left hemisphere is associated with the oral phase and the right hemisphere with the pharyngeal phase. In addition, it was also suggested that damage to a specific hemisphere affects swallowing recovery [7]. Patients with right hemispheric damage were more likely to have persistent dysphagia than patients with left sided lesions [8]. On the other hand, it appeared that subjects with left cerebral infarction complicated by dysphagia, had greater risks of aspiration pneumonia compared to groups with right hemispheric lesions [9].

Several studies revealed that other stroke-related neurological states, such as cognitive dysfunction and neglect were associated with dysphagia $[5,10,11]$. However, most of these studies investigated the relationships between post-stroke dysphagia and cognitive deficits by using simple cognitive screening tests, such as the Mini-Mental State Examination (MMSE) [10]. Although MMSE is commonly used in clinical practices, it does not assess frontal lobe functions and mainly evaluates left hemispheric cognitive functions, including language, verbal memory and calculation [12-14]. Therefore, more comprehensive and domain specific cognitive tests are necessary to investigate the relationships between cognitive deficit and post-stroke dysphagia.

This study has two main purposes (1) to analyze the characteristics of cognitive deficits in patients with subacute post-stroke dysphagia using detailed neuropsy- chological profiles, (2) to investigate the relationships between cognitive deficits and the severity of dysphasia.

\section{MATERIALS AND METHODS}

\section{Participants}

We retrospectively collected and analyzed data of inpatients from the Department of Physical Medicine and Rehabilitation, Korea University Anam Hospital from January 2011 to February 2014. Patients were recruited if they suffered a first-ever stroke of the supratentorial area within 3 months of onset. Patients complaining of dysphagia or showing signs of aspiration performed videofluoroscopic swallowing studies (VFSS). Subjects were excluded if the stroke was not a first-ever episode or an infratentorial lesion. Patients with other neurological conditions, histories of diseases related to swallowing, severe cognitive impairment (MMSE $\leq$ ), or severe aphasia preventing thorough evaluation of neurocognitive function were also excluded. A total of 55 subjects who performed the VFSS and comprehensive cognitive function test were included in this study. The study protocol was approved by the Institutional Review Board of the Korea University Anam Hospital (No. ED13255).

\section{Stroke characteristics}

General characteristics including biographical and neurologic data were collected via chart review. These included the patient's gender, age, education, type of stroke (ischemic or hemorrhagic), brain lesion location (cortical or subcortical), laterality (left, right, or bilateral), interval between stroke onset and VFSS (number of days), and the initial severity of the stroke recorded accordingly to the Korean version of the National Institutes of Health Stroke Scale (K-NIHSS). Functional data including the Fugl-Meyer motor assessment (FMA), Berg Balance Scale (BBS), and the Korean version of the Modified Barthel Index (K-MBI) were also collected.

\section{Assessment of swallowing function}

During VFSS, fluoroscopy was performed as the patients in a seated position swallowed barium mixed with orange juice, with yogurt, with thick gruel, and with rice. Each food type was mixed with undiluted liquid barium for bolus observation during fluoroscopy. In the oral phase, premature bolus loss and oral transit time 
of the bolus were evaluated. In the pharyngeal phase, laryngeal elevation, pharyngeal delay time until the swallowing reflex appeared, pharyngeal transit time of the bolus, aspiration or penetration of the respiratory tract, and pharyngeal post-swallow residues were assessed. Each individual observation was judged on the following parameter cutoffs. Transit time in the oral phase was considered normal if it was less than 1.25 seconds. Pharyngeal delay time, the time until the swallowing reflex appears, was considered normal if less than 0.4 seconds. Normal pharyngeal transit time was considered to be less than 1 second. Penetration was defined as the test material entering the respiratory tract, but not passing into the true vocal fold. If the material passed into the true vocal fold, it was considered to be aspiration. After swallowing, the presence or absence of the pharyngeal post-swallow residue in the vallecular space or the pyriform sinus was also evaluated.

Based on previous studies $[15,16]$, we rated composite scores of the dysphagia severity scale (DSS) based on VFSS findings and the clinical examination, where dysphagia was rated on a scale from 0 (normal) to 4 (severe), depending on delay in the pharyngeal swallow, stasis, and the presence of aspiration.

\section{Comprehensive cognitive function test}

For assessment of cognitive functions, the Korean version of MMSE and the computerized neurocognitive test (CNT; MaxMedica, Seoul, Korea) were performed on every patient within 7 days of the swallowing tests. The following 16 subtests for evaluation of attention, memory, and executive functions were included: digit span test forward and backward, visual span test forward and backward, visual and auditory continuous performance test (CPT), trail making test, Wisconsin Card Sorting Test (WCST), Rey Complex Figure Test and Recognition Trial (RCFT), verbal learning test and Raven Colored Progressive Matrices (RCPM).

Verbal attention and working memory were tested with a digit span test, where the backward test is more specific for working memory [17]. The visual span test tested for visual attention and memory. Visual sustained attention was tested with visual CPT, and auditory sustained attention with auditory CPT [18]. The trail making test was described as a measure of visual attention and perception, sequencing abilities, and executive functions [19]. The
WCST, tested for executive functioning through set shifting, where cards were sorted in accordance with one out of three rules that the patient had to deduce [20]. RCFT was used to test visuospatial constructional abilities and visual memory [21]. The verbal learning test is a wellestablished neuropsychological test for verbal learning and memory [22]. Raven progressive matrices test visual intelligence by showing a series of pictures on the screen and requiring the patient to select the most appropriate picture for a missing space in a series [17].

Subtests with simple instructions and the least verbal commands were selected for the study. All subjects were tested in a quiet room created specifically for evaluation.

We selected a single specific score in every subtest for analysis. For the digital span test and visual span test, the number of digits completed was taken. For auditory CPT and visual $\mathrm{CPT}$, the number of correct responses was selected. For trail making tests A/B, the time taken to complete the set was analyzed. For the WCST, the number of categories completed by the patient was used. For RCFT, the score for accurate reproduction and placement of the 18 specific design elements was used. For the verbal learning test, the number of list elements recalled was analyzed. For Raven progressive matrices, the number of correct responses was used.

\section{Statistical analysis}

SPSS ver. 20 (IBM, Armonk, NY, USA) software was used for statistical analysis. We analyzed the differences in demographic and clinical characteristics between the two groups using a $\chi^{2}$ test and a Mann-Whitney test. Variables indicating a significant difference in demographic and clinical characteristics were regarded as confounding variables and used to calculate correlation coefficients for any correlation with degree of dysphagia and the parameters of swallowing phases. To investigate the differences in cognitive performance between the two groups, we performed a Mann-Whitney test. To look for any correlations between cognitive deficits and degrees of dysphasia, Spearman partial correlation coefficients were calculated between DSS and the subtest results of CNT. Multivariate logistic regression analysis was performed to assess relationships among stroke performance scores, neuropsychological profiles, and the presence or absence of dysphagia. The relationships among parameters of swallowing phases and the results of each cognitive 
subtest were also calculated using a multivariate logistic regression analysis. A p-value of less than 0.05 was considered to be significant.

\section{RESULTS}

Among all 55 subjects, 32 cases were included in the non-dysphagia group, and 23 cases were included in the dysphagia group.

\section{Differences between normal and dysphagia groups \\ Clinical characteristics of stroke}

Clinical characteristics of participants are summarized in Table 1. Biographical and neurological factors showed no significant differences. Stroke type, lesion location, and laterality showed no associations with the presence of dysphagia. FMA, BBS, and K-MBI showed significantly lower scores in the dysphagia group compared to the non-dysphagia group, suggesting that the dysphagia group had more severe functional impairments in motor, balance, and ADL compared to the non-dysphagia group.

\section{Results of cognitive function tests}

Results of CNT are shown in Table 2. The non-dysphagia group showed better performance than the dysphagia group in all cognitive tests. The statistically significant subtests were as follows: visual span test forward $(\mathrm{p}=0.012)$ and backward $(\mathrm{p}=0.003)$; visual CPT $(\mathrm{p}=0.018)$; the RCFT immediate recall ( $\mathrm{p}=0.004)$; WCST $(\mathrm{p}=0.040)$; and trail making test $A(p=0.017)$. In addition, the scores of RCPM ( $\mathrm{p}=0.009)$ were significantly higher in the nondysphagia group. RCFT copy $(\mathrm{p}=0.050)$ and RCFT delayed

Table 1. Demographic and clinical characteristics of patient subgroups $(\mathrm{n}=55)$

\begin{tabular}{|c|c|c|c|}
\hline Characteristic & Non-dysphagia $(n=32)$ & Dysphagia $(n=23)$ & p-value \\
\hline Sex & & & $0.911^{\mathrm{a})}$ \\
\hline Male & 19 & 14 & \\
\hline Female & 13 & 9 & \\
\hline Age (yr) & $57.75 \pm 14.424$ & $58.48 \pm 12.277$ & $0.746^{\mathrm{b})}$ \\
\hline Education (yr) & $9.91 \pm 4.589$ & $9.13 \pm 5.587$ & $0.806^{\mathrm{b})}$ \\
\hline Stroke type & & & $0.732^{\mathrm{a})}$ \\
\hline Ischemic & 18 & 14 & \\
\hline Hemorrhagic & 14 & 9 & \\
\hline Location & & & $0.338^{\mathrm{a})}$ \\
\hline Cortical & 9 & 3 & \\
\hline Subcortical & 19 & 15 & \\
\hline Laterality & & & $0.594^{\mathrm{a})}$ \\
\hline Left & 14 & 7 & \\
\hline Right & 4 & 12 & \\
\hline Bilateral & 4 & 4 & \\
\hline Interval between onset of stroke and VFSS (day) & $44.44 \pm 53.658$ & $42.61 \pm 46.925$ & $0.932^{\mathrm{b})}$ \\
\hline K-NIHSS & $6.22 \pm 2.915$ & $7.26 \pm 3.306$ & $0.204^{\mathrm{b})}$ \\
\hline K-MMSE & $22.03 \pm 5.451$ & $19.74 \pm 6.621$ & $0.208^{\mathrm{b})}$ \\
\hline Fugl-Meyer & $56.13 \pm 31.434$ & $37.61 \pm 31.985$ & $0.024^{* \mathrm{~b})}$ \\
\hline BBS & $27.59 \pm 18.684$ & $14.09 \pm 16.332$ & $0.002^{* * b)}$ \\
\hline K-MBI & $56.38 \pm 23.194$ & $40.78 \pm 24.110$ & $0.022^{* b)}$ \\
\hline
\end{tabular}

Values are represented as number or mean \pm standard deviation.

VFSS, videofluoroscopic swallowing study; K-NIHSS, Korean version of National Institutes of Health Stroke Scale; KMMSE, Korean version of Mini-Mental Status Examination; Fugl-Meyer, Fugl-Meyer motor assessment; BBS, Berg Balance Scale; K-MBI, Korean version of Modified Barthel Index.

${ }^{*} \mathrm{p}<0.05,{ }^{* *} \mathrm{p}<0.01$ (difference between non-dysphagia group and dysphagia group).

${ }^{\text {a) }}$ Mann-Whitney U-test, ${ }^{\text {b) }} \chi^{2}$-test. 
Table 2. Comparison of cognitive battery mean scores of the two subgroups $(\mathrm{n}=55)$

\begin{tabular}{|c|c|c|c|}
\hline Subtest & $\begin{array}{c}\text { Non- } \\
\text { dysphagia } \\
(\mathrm{n}=\mathbf{3 2})\end{array}$ & $\begin{array}{c}\text { Dysphagia } \\
(\mathbf{n}=\mathbf{2 3})\end{array}$ & p-value \\
\hline \multicolumn{4}{|l|}{ Digit span } \\
\hline Forward (8) & 4.45 & 4.05 & 0.726 \\
\hline Backward (7) & 2.45 & 2.05 & 0.355 \\
\hline \multicolumn{4}{|l|}{ Visual span } \\
\hline Forward (8) & 3.75 & 2.78 & $0.012^{*}$ \\
\hline Backward (7) & 3.09 & 1.78 & $0.003^{* *}$ \\
\hline Auditory CPT (135) & 89.63 & 69.00 & 0.13 \\
\hline Visual CPT (135) & 104.75 & 86.48 & $0.018^{*}$ \\
\hline \multicolumn{4}{|l|}{ RCFT } \\
\hline Copy (36) & 22.633 & 16.304 & 0.050 \\
\hline Immediate recall (36) & 10.950 & 4.848 & $0.004^{* *}$ \\
\hline Delayed recall (36) & 8.933 & 4.652 & 0.055 \\
\hline \multicolumn{4}{|l|}{ Verbal learning } \\
\hline Al (15) & 3.59 & 3.25 & 0.813 \\
\hline A5 (15) & 7.03 & 5.95 & 0.374 \\
\hline Delayed (15) & 4.10 & 3.10 & 0.486 \\
\hline Card sorting test (6) & 3.17 & 1.38 & $0.040^{*}$ \\
\hline Trail making test A (s) & 93.58 & 159.50 & $0.017^{*}$ \\
\hline Trail making test $\mathrm{B}(\mathrm{s})$ & 143.50 & 265.00 & 0.314 \\
\hline $\begin{array}{l}\text { Raven progressive } \\
\text { matrices (36) }\end{array}$ & 17.03 & 10.09 & $0.009^{* *}$ \\
\hline
\end{tabular}

The numbers in parentheses are maximum score.

CPT, continuous performance test; RCFT, Rey Complex Figure Test and Recognition Trial.

${ }^{*} \mathrm{p}<0.05,{ }^{* *} \mathrm{p}<0.01$ (between non-dysphagia group and dysphagia group by Mann-Whitney test).

recall ( $\mathrm{p}=0.055)$ scores exhibited a borderline significant difference between the two groups.

\section{Correlation between dysphagia severity and cognitive tests}

Correlation coefficients calculated between DSS and cognitive subtests are shown in Table 3. Subtests showing significant correlations with DSS were trail making test $\mathrm{A}(\mathrm{p}=0.042)$ trail making test $\mathrm{B}(\mathrm{p}=0.002)$, and $\mathrm{RCPM}$ $(\mathrm{p}=0.002)$ after adjusting by age, FMA, BBS, and K-MBI. Other subtests failed to show significant correlations.

Factors associated with dysphagia and parameters of oral and pharyngeal phase

We investigated clinical or neuropsychological factors
Table 3. Correlation with scores of dysphagia severity

\begin{tabular}{|lcl|}
\hline \multicolumn{1}{|c}{ Subtest } & $\begin{array}{c}\text { Correlation } \\
\text { coefficient }\end{array}$ & p-value \\
\hline Digit span & & \\
\hline Forward (8) & 0.163 & 0.307 \\
\hline Backward (7) & -0.051 & 0.751 \\
\hline Visual span & & \\
\hline Forward (8) & -0.216 & 0.174 \\
\hline Backward (7) & -0.324 & $0.039^{*}$ \\
\hline Auditory CPT (135) & -0.201 & 0.207 \\
\hline Visual CPT (135) & -0.240 & 0.131 \\
\hline RCFT & & \\
\hline Copy (36) & -0.260 & 0.101 \\
\hline Immediate recall (36) & -0.349 & 0.025 \\
\hline Delayed recall (36) & -0.161 & 0.313 \\
\hline Verbal learning & & \\
\hline A1 (15) & -0.051 & 0.751 \\
\hline A5 (15) & -0.153 & 0.339 \\
\hline Delayed (15) & -0.212 & 0.183 \\
\hline Card sorting test (6) & -0.215 & 0.178 \\
\hline Trail making test A (s) & -0.319 & $0.042^{*}$ \\
\hline Trail making test B (s) & -0.462 & $0.002^{* *}$ \\
\hline Raven progressive matrices (36) & -0.471 & $0.002^{* *}$ \\
\hline
\end{tabular}

The numbers in parentheses are maximum score.

CPT, continuous performance test; RCFT, Rey Complex Figure Test and Recognition Trial.

${ }^{*} p<0.05,{ }^{* *} p<0.01$ of Spearman partial correlation coefficient adjusted by age, Fugl-Meyer motor assessment, Berg Balance Scale, and the Korean version of the Modified Barthel Index.

determining the presence of dysphagia using a logistic regression analysis. The results are summarized in Table 4. Univariate analysis indicated significant differences for FMA, BBS, K-MBI, visual span test, both forward and backward, auditory CPT, RCFT, WCST, and RCPM $(\mathrm{p}<0.05)$. When a dependent variable was set as a binary outcome by the presence of dysphagia, multivariate binary logistic regression analysis identified 2 independent predictors: FMA (odds ratio $[\mathrm{OR}]=0.972 ; 95 \%$ confidence interval [CI], 0.947-0.982; $\mathrm{p}=0.036$ ), and trail making test B (OR=1.006; 95\% CI, 1.002-1.009; $p=0.001)$. No significant associations were observed for other variables. The $\mathrm{R}^{2}$ of the model was 0.385 .

Multivariate binary logistic regression analyses for parameters of oral and pharyngeal phase were also executed. Trail making test B (OR=1.009; 95\% CI, 1.002-1.016; 
Table 4. Factors associated with the presence of dysphagia

\begin{tabular}{|c|c|c|c|c|}
\hline Variable & Unadjusted OR (95\% CI) & p-value a) $^{\text {a }}$ & Adjusted OR (95\% CI) & p-value $e^{\text {b) }}$ \\
\hline Fugl-Meyer & $0.982(0.964-0.999)$ & $0.041^{*}$ & $0.972(0.947-0.998)$ & $0.036^{*}$ \\
\hline BBS & $0.957(0.926-0.990)$ & $0.011^{*}$ & & \\
\hline K-MBI & $0.972(0.949-0.996)$ & $0.024^{*}$ & & \\
\hline \multicolumn{5}{|l|}{ Visual span } \\
\hline Forward (8) & $0.592(0.383-0.916)$ & $0.019^{*}$ & & \\
\hline Backward (7) & $0.531(0.343-0.821)$ & $0.004^{* *}$ & & \\
\hline Auditory CPT (135) & $0.980(0.963-0.996)$ & $0.018^{*}$ & & \\
\hline Trail making test B (s) & $1.005(1.002-1.008)$ & $0.002^{* *}$ & $1.006(1.002-1.009)$ & $0.001^{* *}$ \\
\hline \multicolumn{5}{|l|}{ RCFT } \\
\hline Сopy (36) & $0.952(0.908-0.997)$ & $0.039^{*}$ & & \\
\hline Immediate recall (36) & $0.883(0.805-0.967)$ & $0.007^{* *}$ & & \\
\hline Delayed recall (36) & $0.909(0.830-0.995)$ & $0.040^{*}$ & & \\
\hline Card sorting test (6) & $0.701(0.511-0.960)$ & $0.027^{*}$ & & \\
\hline Raven progressive matrices (36) & $0.916(0.857-0.980)$ & $0.011^{*}$ & & \\
\hline
\end{tabular}

The numbers in parentheses are maximum score.

OR, odds ratio; CI, confidence interval; Fugl-Meyer, Fugl-Meyer motor assessment; BBS, Berg Balance Scale; K-MBI, Korean version of Modified Barthel Index; CPT, continuous performance test; RCFT, Rey Complex Figure Test and Recognition Trial.

${ }^{*} \mathrm{p}<0.05,{ }^{* *} \mathrm{p}<0.01$.

${ }^{a}$ Unadjusted odds ratios by logistic regression analysis. ${ }^{\text {b) }}$ Adjusted odds ratios by multivariate logistic regression analysis of variables which proved to have a significant relationship with dysphagia.

$\mathrm{p}=0.017$ ) was the only significant risk factor for premature loss. The $\mathrm{R}^{2}$ of the model was 0.653 . There were no significant predictors for oral transit time, pharyngeal transit time, pharyngeal delay time, and post-swallow residue proven by logistic regression.

\section{DISCUSSION}

The aim of this study was to investigate characteristics of the post-stroke dysphagia group and to determine the relationships between swallowing dysfunction and cognitive functions in patients with supratentorial stroke. Our results indicate that the dysphagia group showed significantly lower scores in motor, balance, and ADL function than the non-dysphagia group, coinciding with previous studies $[23,24]$. Cognitive function tests revealed lower performance in all measured subtests for the dysphagia group compared to the non-dysphagia group. Dysphagia severity was significantly correlated with the visual span test, backward, the trail making tests A and B (each representing visual attention and executive function), and the RCPM test, representing visuospatial intelligence. Param- eters of oral phase, especially premature loss, were also significantly correlated with cognitive subtests, notably for visual attention and executive function. Overall, swallowing ability was significantly correlated with subsets of cognitive functions related to visual cues rather than functions requiring auditory attention or verbal memory function.

Recent neuroimaging studies have investigated the brain regions associated with swallowing. Gonzale et al. [25] proposed the basal ganglia, putamen, caudate, internal capsule and somatosensory related cortex area (primary somatosensory, motor, and motor supplementary area) as possible candidates. Martin et al. [26] investigated the cerebral cortical region activated during automatic and volitional swallowing. They found that the lateral precentral gyrus, lateral postcentral gyrus, right insula, superior temporal gyrus, middle and inferior frontal gyri, and frontal operculum were activated during all swallowing tasks. These findings and the study of swallowing lateralization [6] suggest different contributions of left and right hemispheres, but we could not find differences in swallowing function between patients with left 
and right hemispheric lesions in this study. According to these findings, swallowing behavior is controlled not only by the brainstem, but also by higher centers, including the frontal cerebral cortex and subcortex [27]. Swallowing sequences require more complex cascades including cognitive processes and are not just reflexive [28].

There have been few studies that investigated the association of dysphagia with cognitive impairment in patients after stroke. Earlier work for example revealed that low MMSE scores and neglect were associated with dysphagia $[10,11]$. However, there are no studies specifically addressing the relationship of components of cognitive functioning assessed by comprehensive neuropsychological profiles, and swallowing function after a stroke. Some reports have described the association between cognitive and swallowing functions, but these were performed with healthy subjects or on patients with other brain disorders. In healthy participants, disruptive stimuli might alter feeding, but had little effect on oropharyngeal swallowing [29]. A study in Parkinson's disease patients suggested that there are significant correlations between frontal/executive or learning/memory functions and the oral phase of swallowing, whereas the pharyngeal phase showed weak correlations with frontal functions [30]. In Alzheimer's disease (AD), dysphagia has also been thought to be associated with cognitive impairment [31]. If cortical involvement and cognitive decline progressed, swallowing problems could worsen in $\mathrm{AD}[31,32]$.

The oral phase of swallowing was reported to correlate with attention and executive functions in $\mathrm{AD}$ patients [31]. Our study also confirmed that visual attention and frontal executive functions significantly correlated with dysphagia severity in subacute stroke patients. Therefore, we can assume that it is essential to evaluate swallowing functions for stroke patients with deficits of attention or executive functioning, to understand the underlying mechanism of dysphagia and to set up proper dysphagia rehabilitation programs.

This study has some limitations. First, the number of subjects was not large enough to properly assess the associations of swallowing function with other clinical data. A larger sample could have led to more significant findings and revealed stronger relationships between neuropsychological profiles and swallowing. Second, only inpatients were recruited, so there may have been a selection bias. Third, our study was retrospectively de- signed, and therefore, the results should be treated with caution and interpreted carefully in aspects of causality. Additionally, we did not evaluate brain anatomy in detail using imaging techniques. Functional imaging studies or tractography could provide valuable information about the relationships between the two functions. Despite these limitations, we found correlations between cognitive functioning, especially visual attention and executive functions, and swallowing performance in supratentorial stroke patients. Therefore, this report represents a meaningful pilot study for that cause. We can assume that it is important to adequately evaluate swallowing functions in stroke patients with cognitive impairments. Additionally, cognitive rehabilitation, especially for attention and frontal lobe function, could have positive impacts on swallowing performance, notably for problems with the oral phase. Further studies on the effect of cognitive rehabilitation for recovery of swallowing functions may be meaningful. A prospectively designed study with a larger sample size including healthy controls is necessary to investigate more clearly the relationships between cognitive functions and swallowing. Serial follow-up measurements for cognitive functioning and dysphagia recovery will also provide further valuable information.

In conclusion, our study suggests that cognitive functions might contribute to the severity of dysphagia in stroke patients. Visual attention and executive function may influence the oral phase of swallowing in patients with supratentorial lesions. Therefore, detection and treatment for coexisting cognitive impairment may be helpful or even crucial for efficient dysphagia treatment and successful rehabilitation.

\section{CONFLICT OF INTEREST}

No potential conflict of interest relevant to this article was reported.

\section{ACKNOWLEDGMENTS}

This research was supported by grant of Korea Research Foundation (No. NRF-2016R1A2B4009206).

\section{REFERENCES}

1. Gordon C, Hewer RL, Wade DT. Dysphagia in acute 
stroke. Br Med J (Clin Res Ed) 1987;295:411-4.

2. Splaingard ML, Hutchins B, Sulton LD, Chaudhuri G. Aspiration in rehabilitation patients: videofluoroscopy vs bedside clinical assessment. Arch Phys Med Rehabil 1988;69:637-40.

3. Robbins J, Levin RL. Swallowing after unilateral stroke of the cerebral cortex: preliminary experience. Dysphagia 1988;3:11-7.

4. Alberts MJ, Horner J, Gray L, Brazer SR. Aspiration after stroke: lesion analysis by brain MRI. Dysphagia 1992;7:170-3.

5. Robbins J, Levine RL, Maser A, Rosenbek JC, Kempster GB. Swallowing after unilateral stroke of the cerebral cortex. Arch Phys Med Rehabil 1993;74:1295-300.

6. Daniels SK, Corey DM, Fraychinaud A, DePolo A, Foundas AL. Swallowing lateralization: the effects of modified dual-task interference. Dysphagia 2006;21: 21-7.

7. Daniels SK, Foundas AL. Lesion localization in acute stroke patients with risk of aspiration. J Neuroimaging 1999;9:91-8.

8. Smithard DG, O'Neill PA, Martin DF, England R. Aspiration following stroke: is it related to the side of the stroke? Clin Rehabil 1997;11:73-6.

9. Yamamoto K, Koh H, Shimada H, Takeuchi J, Yamakawa Y, Kawamura M, et al. Cerebral infarction in the left hemisphere compared with the right hemisphere increases the risk of aspiration pneumonia. Osaka City Med J 2014;60:81-6.

10. Schroeder MF, Daniels SK, McClain M, Corey DM, Foundas AL. Clinical and cognitive predictors of swallowing recovery in stroke. J Rehabil Res Dev 2006;43: 301-10.

11. Moon HI, Pyun SB, Kwon HK. Correlation between location of brain lesion and cognitive function and findings of videofluoroscopic swallowing study. Ann Rehabil Med 2012;36:347-55.

12. Tombaugh TN, McIntyre NJ. The mini-mental state examination: a comprehensive review. J Am Geriatr Soc 1992;40:922-35.

13. Aggarwal A, Kean E. Comparison of the Folstein Mini Mental State Examination (MMSE) to the Montreal Cognitive Assessment (MoCA) as a cognitive screening tool in an inpatient rehabilitation setting. Neurosci Med 2010;1:39-42.

14. Feher EP, Mahurin RK, Doody RS, Cooke N, Sims J,
Pirozzolo FJ. Establishing the limits of the Mini-Mental State: examination of 'subtests'. Arch Neurol 1992; 49:87-92.

15. Daniels SK, Brailey K, Priestly DH, Herrington LR, Weisberg LA, Foundas AL. Aspiration in patients with acute stroke. Arch Phys Med Rehabil 1998;79:14-9.

16. Daniels SK, McAdam CP, Brailey K, Foundas AL. Clinical assessment of swallowing and prediction of dysphagia severity. Am J Speech Lang Pathol 1997;6:1724.

17. Unterrainer JM, Rahm B, Kaller CP, Leonhart R, Quiske K, Hoppe-Seyler K, et al. Planning abilities and the Tower of London: is this task measuring a discrete cognitive function? J Clin Exp Neuropsychol 2004;26: 846-56.

18. Tinius TP. The Integrated Visual and Auditory Continuous Performance Test as a neuropsychological measure. Arch Clin Neuropsychol 2003;18:439-54.

19. Salthouse TA. What cognitive abilities are involved in trail-making performance? Intelligence 2011;39:22232.

20. Monchi O, Petrides M, Petre V, Worsley K, Dagher A. Wisconsin Card Sorting revisited: distinct neural circuits participating in different stages of the task identified by event-related functional magnetic resonance imaging. J Neurosci 2001;21:7733-41.

21. Shin MS, Park SY, Park SR, Seol SH, Kwon JS. Clinical and empirical applications of the Rey-Osterrieth Complex Figure Test. Nat Protoc 2006;1:892-9.

22. Schoenberg MR, Dawson KA, Duff K, Patton D, Scott JG, Adams RL. Test performance and classification statistics for the Rey Auditory Verbal Learning Test in selected clinical samples. Arch Clin Neuropsychol 2006;21:693-703.

23. Kidd D, Lawson J, Nesbitt R, MacMahon J. Aspiration in acute stroke: a clinical study with videofluoroscopy. Q J Med 1993;86:825-9.

24. Nilsson H, Ekberg O, Olsson R, Hindfelt B. Dysphagia in stroke: a prospective study of quantitative aspects of swallowing in dysphagic patients. Dysphagia 1998; 13:32-8.

25. Gonzalez-Fernandez M, Kleinman JT, Ky PK, Palmer JB, Hillis AE. Supratentorial regions of acute ischemia associated with clinically important swallowing disorders: a pilot study. Stroke 2008;39:3022-8.

26. Martin RE, Goodyear BG, Gati JS, Menon RS. Cerebral 
cortical representation of automatic and volitional swallowing in humans. J Neurophysiol 2001;85:93850.

27. Hamdy S, Aziz Q, Rothwell JC, Crone R, Hughes D, Tallis RC, et al. Explaining oropharyngeal dysphagia after unilateral hemispheric stroke. Lancet 1997;350:68692.

28. Patti F, Emmi N, Restivo DA, Liberto A, Pappalardo A, Torre LM, et al. Neurogenic dysphagia: physiology, physiopathology and rehabilitative treatment. Clin Ter 2002;153:403-19.

29. Brodsky MB, McNeil MR, Martin-Harris B, Palmer CV,
Grayhack JP, Abbott KV. Effects of divided attention on swallowing in healthy participants. Dysphagia 2012; 27:307-17.

30. Kim JS, Youn J, Suh MK, Kim TE, Chin J, Park S, et al. Cognitive and motor aspects of parkinson's disease associated with dysphagia. Can J Neurol Sci 2015;42: 395-400.

31. Secil Y, Arici S, Incesu TK, Gurgor N, Beckmann Y, Ertekin C. Dysphagia in Alzheimer's disease. Neurophysiol Clin 2016;46:171-8.

32. Chouinard J. Dysphagia in Alzheimer disease: a review. J Nutr Health Aging 2000;4:214-7. 